\title{
The Influence of Behavioral Finance on Capital Market in China - Take the Asymmetrical Stock Market Fluctuation as an Example
}

\author{
Yiqin Sun \\ School of Economics, Shanghai University, Shanghai, China
}

Email address:

jenny_sun@shu.edu.cn

\section{To cite this article:}

Yiqin Sun. The Influence of Behavioral Finance on Capital Market in China - Take the Asymmetrical Stock Market Fluctuation as an Example. Science Innovation. Vol. 6, No. 4, 2018, pp. 232-239. doi: 10.11648/j.si.20180604.20

Received: June 25, 2018; Accepted: July 30, 2018; Published: August 3, 2018

\begin{abstract}
The volatility of stock returns is affected by macro variables, economic policies, company fundamentals and investor sentiment. This paper studies the effect of behavioral finance on the asymmetry of stock market volatility, and selects the CSI 300 daily return rate from January 4, 2008 to December 31, 2016 as the research observations, using the EGARCH model that introduces the loss aversion function to model.The CSI 300 index daily returns volatility model is empirically tested. Empirical findings: (1) Loss of aversion and overreaction can explain the asymmetry (leverage) of volatility in China's stock market, that is, the fluctuation caused by the same amount of "bad" is greater than the fluctuation caused by the same "good" news. (2) The revised model more closely describes the asymmetry of China's stock market volatility and reflects the impact of asymmetric volatility on stock market risk control, pricing, and asset allocation. Finally, the article proposes to adopt a reversal strategy to reduce the bias caused by some human factors by quantifying transactions.
\end{abstract}

Keywords: Stock Market Volatility, Asymmetry, Loss Aversion, Overreaction

\section{行为金融对资本市场的影响一以股市波动非对称为例}

\section{孙怡沁}

经济学院, 上海大学, 上海, 中国

\section{邮箱:}

jenny_sun@shu.edu.cn

摘要: 股票收益率波动受到宏观变量、经济政策、公司基本面状况和投资者情绪的影响。本文研究行为金融学对股市 波动率非对称的影响, 选取2008年1月4日至2016年12月31日的沪深300指数日收益率为研究样本, 将损失厌恶函数引入 EGARCH模型, 对沪深300指数日收益率波动率建模进行实证检验。实证发现: (1)损失戻恶和过度反应能解释中国股 市波动的非对称性（杜杆性），即等量的“利空”带来的波动大于等量“利好”消息带来的波动。(2)修改后的模型更贴近 实际地刻画了中国股市波动的非对称性，体现非对称波动对股市风控、定价、资产配置的影响。最后文章提出采取反 转策略，通过量化交易减少部分人为因素导致的偏差等建议。

关键词: 股市波动率, 非对称, 损失厌恶, 过度反应 


\section{1. 引言}

股票收益率波动受到宏观变量（如市场流动性）、公 司基本面和投资者情绪的综合影响。中国股票市场历史较 短, 与西方发达国家股市相比, 中国股市的波动率相对较 高 [3]。同时中国股市个人投资者比例较高, 基于个人的 心理偏差和行为偏差会对股市的波动率产生较大影响, 以 至于会影响股市的定价、管理风险、配置资产的作用, 使 得股市的财富效应、q效应等难以发挥, 也阻碍了股市与 实体经济的良性互动。因此研究投资者情绪对股市波动率 的作用机制，以及投资者情绪造成的影响后果十分重要， 这有助于投资者更好地理解市场, 把握市场; 以及监管层 面更好地管控调节。

行为金融学诞生至今从理论上对投资者情绪和投资 行为进行了详细解释和刻画, 因此本文基于行为金融学的 理论探讨投资者情绪和投资者特质。其中，行为金融学的 重要理论一一前景理论认为投资者对信息产生不同反应, 面对同样数量的收益和损失时, 认为损失更令人难受, 这 种认识偏差被称为损失厌恶。同时投资者对于损失的信息 过度看重会导致过度反应, 即同等程度的利好和利空信息 对股价的影响不同，即存在波动的非对称性。

本文将行为金融学中的价值函数中的损失厌恶描述 部分引入EGARCH模型, 以投资者损失厌恶、过度反应为 解释变量, 建立新的模型, 新模型更贴近实际地刻画了中 国股市波动的非对称性, 这种新模型为研究股票市场波动 提供了一种新视角——投资者情绪和投资者特征角度。

\section{2. 文献综述}

\section{1. 收益波动率的刻画}

学界和业界多数采用时间序列模型对波动率进行刻 画。其中采用的最多的是ARMA模型与 $\mathrm{ARCH} 、 \mathrm{GARCH}$ 族模型。Box和Jenkins[26]在 20 世纪70年代提出ARMA模 型, 用单序列过去状态解释当前。ARMA模型的基础是自 回归模型 $\mathrm{AR}(\mathrm{p})$ 和移动平均模型 $M A(p)$ 。

自回归模型 $A R(p)$ :

$$
\mathrm{X}_{\mathrm{t}}=\varepsilon_{\mathrm{t}}+\theta_{1} \varepsilon_{\mathrm{t}-1}+\ldots \ldots+\mu_{\mathrm{t}}
$$

移动平均模型 $M A(p)$ :

$$
X_{\mathrm{t}}=\phi_{0}+\phi_{1} X_{\mathrm{t}-1}+\ldots \ldots+\varepsilon_{\mathrm{t}}
$$

自回归移动平均模型 $\operatorname{ARMA}(\mathrm{p}, \mathrm{q})$ :

$$
X_{t}=\phi_{0}+\sum_{i=1}^{p} \phi_{1} X_{t-i}+\varepsilon_{t}-\sum_{i=1}^{p} \theta_{i} \varepsilon_{t-i}
$$

郑伟伦（2014）[8]基于ARIMA模型对上证综合指数 股票价格短期预测。Engle（1982）[27]首次提出自回归条 件异方差模型，指出金融时间序列条件方差会随着时间变 化, 可以看成是滞后项 $\mu$ 的函数, 收益的波动率受过去信
息的影响。均值方程就是ARMA的相关方程。条件异方差 方程为 $\mathrm{ARCH}(\mathrm{q})$ :

$$
\sigma_{\mathrm{t}}^{2}=\mathrm{a}_{0}+\mathrm{a}_{1} \mu_{\mathrm{t}-1}^{2}+\lambda_{1} \sigma_{\mathrm{t}-1}^{2}
$$

Bollerslev (1986) [25]进行了扩展, 同时解决了ARCH 模型中存在的一些问题, 使得待估参数数量减少, 提高了 模型估计的准确度, 形成广义自回归条件异方差GARCH 模型, 之后产生了 GARCH族模型。GARCH模型与ARCH 模型的不同之处在于模型中增加了 $\mathrm{q}$ 个自回归项目, 即 GARCH模型是一个高阶的ARCH模型。

随着GARCH模型在金融领域的广泛运用, 学者发现 基础的 GARCH函数不能很好地反映实际金融资产运动中 所存在的杜杆效应，理论上金融时间序列条件方差是滞后 项 $\mu$ 的对称函数, 而实际中往往是不对称的, 为了解决以 上问题, Nelson(1991)[12]提出了指数GARCH(E-GARCH) 模型来捕获和提取这种不对称性, 从而能更贴近实际刻画 股票的波动性。

其中EGRACH可研究非对称效应, 其中条件异方差方 程为:

$$
\ln \left(\sigma_{\mathrm{t}}{ }^{2}\right)=\omega+\beta \ln \left(\sigma_{\mathrm{t}-1}{ }^{2}\right)+\alpha\left|\frac{\mu_{\mathrm{t}-1}}{\sigma_{\mathrm{t}-1}}-\sqrt{\frac{2}{\pi}}\right|+\gamma \frac{\mu_{\mathrm{t}-1}}{\sigma_{\mathrm{t}-1}}
$$

$\omega 、 \beta 、 \alpha 、 \gamma$ 分别为EGARCH模型常数项系数、 GARCH项系数、ARCH项系数和杜杆效应系数。

$\gamma<0$ 时存在波动杜杆, 等量利空 (坏消息) 带来波 动大于利好信息 (好消息) 带来的波动。

\section{2. 波动率不对称性的刻画}

波动率不对称性效应、也称为“杜杆效应”(leverage Effect)最早由Black(1976)、Christie(1982)和Schwert(1989) [12]提出, 指的是等量利空消息比利好消息带来的波动更 大的“杜杆效应”。即等量利空消息带来波动大于利好的杜 杆效应指的是利空消息将会引发股价下跌, 从而增大公司 财务杜杆, 导致价格波动增大。以往理论从宏观、政策、 市场关联等解释股市波动。Morelli (2002) 发现汇率波动 可显著解释股市波动。Schwarz（2006）研究美国股市发 现货币供应量与股票市场之间存在显著关系。 Jeon(1990)[12]指出市场联动效应导致了股价过度波动。排 除这些因素, 本文将考虑投资者情绪导致的波动利率变化。

西方学者对各国股市波动的非对称性研究很多, 如 Koumos和Booth(1995) 研究了日本、伦敦、纽约股市收益 的潜在非对称性的波动。Crouhy和 Rockinger(1997)用 AT-GARCH和HGARCH模型对全球 21 个主要的股市的波 动性进行了实证检验, 发现确实存在波动的非对称性。 Cheung(1992)、Cathy.W.S等学者发现美国市场也有这种情 况。Poon和Taylor(1992)[12]发现英国、法国、加拿大也有 这种不对称的反应。Chiang 和 Dong (2001) [12] 用 TAR-GARCH模型验证了亚洲七个股票交易所的情况。 Spyros（2012）[36]发现用时间序列相关模型发现 
NYSE,AMEX和NASDAQ指数都有波动不对称的特性。 Sayim 和Morris(2013) [34]用类似方法证明伊斯坦布尔股 票市场指数的波动不对称。Uygur和Taş（2014）[38]用 EGARCH模型实证发现美国, 日本，香港，英国，法国， 德国和土耳其的市场指数也收到不对称的冲击。

中国这近年也有许多学者对股价波动的非对称性效 应也进行了研究。陆荣、徐龙炳 (2003) 何晓光 (2005)、 张路胶、赵华 (2006) [12]等学者采用, ARCH、ARMA、 GARCH族模型对中国股市进行了实证检验。刘璐等 (2011) [10]基于GARCH族模型对亚洲地区股票指数收益率波动 性进行研究。朱钧钧和谢识予（2011）[20]基于 MS-TGARCH模型的MCMC估计和分析中国股市波动率 的双重不对称性。陆静和周媛用GARCH族模型（2015） [13]发现国大陆股票市场的波动不对称性受影响的持续时 间更长。翟佳茹（2017）[16]基于GRACH类模型研究了的 法兰克福DAX指数收益率波动性不对称情况。

综合国内和国外的研究, 我们发现多数相关研究还是 着重证明或者检验了股市波动的非对称性是否存在, 深入 研究股市波动性存在的原因、波动带来影响、作用机制, 以及如何应对股市波动性的文献和实证相对较少。

\section{3. 行为金融学影响市场波动}

过去中国学者和西方国家学者往往着重考察投资者 情绪对市场收益的影响, 而较少关注情绪影响市场波动方 面的关系。近年来, 随着行为金融学, 尤其是前景理论的 发展, 越来越多的学者借助前景理论的某些理论, 进一步 对投资者情绪、投资者特质对股市波动性的影响作用机理 进行了解释。

Kahneman和Tversky（1979）提出的前景理论，有效 突破了期望效用理论 (Expected utility theory) 建立了价值 函数, 更好地描述了人的真实决策偏好特征。这种决策偏 好在经济意义上表现为: 面对盈利时风险厌恶和面对损失 时风险喜好, 尤其是对损失的厌恶超过了对同等收益的喜 好。表现在人们面临收益时愿意保守, 面临损失时愿意冒 险。认为投资者在信息以及认知偏差的影响下在股市中表 现出各种各样的心理和行为特征, 如损失厌恶、后悔、过 度自信、过度反应等也会影响到股市波动率。卢斌(2008)

[12]采用EGARCH、TARCH模型, 研究损失庈恶与反应过 度对上证综指和深证综指日收益率的波动性不对称的影 响。杨阳等 (2010) [3]、胡昌生 (2013) [9]、张宗新 (2013) [18]发现投资者情绪对预期收益波动的冲击存在非对称效 应，牛市股票收益与投资者情绪正相关，而熊市负相关。 文凤华等（2014）[14]用EGARCH模型提取到达市场上的 信息流作为财富改变的代理变量进行了进一步的研究。德 国学者Schneller、Heiden和Heiden (2017) [36]在时间序列 中加入经验相似性 (ES) 方法发现投资者情绪是影响德国 股市波动的重要因素。Baek（2016）[22]发现极端情绪对 股市波动的影响。贺刚等 (2015) [7]发现中国股市的确存
在明显的“创造空间效应”和“弗里德曼效应”这两投资者 情绪效应对中国股市收益的波动非对称产生影响。巴曙松， 朱虹（2016）[1]发现发现投资者情绪对内地A股市场的加 剧波动影响程度最大。朱超和曹洳宜（2017）[21]研究发 现投资者非理性情绪、分析师评级变动影响股票市场波动。 邵振文和侯丹（2017）[4]将投资者情绪引入期货市场, 解 释了我国股指期货市场非对称性波动与下行风险的成因。 单馨等 (2017) [5]发现投资者情绪与不同规模股票市场指 数的不对称波动有显著相关关系。张普等 (2018) [17]研 究了投资者在信息视角下基于异质信念等因认知偏差导 致的对股票波动性影响。

之后, 更多的学者从情绪对股市波动率影响的不同理 论出发, 构建不同指标, 进行实证检验。Yang (2016) [41] 研究了投资者情绪度对短期股票市场（2015年)中国股灾 期间）的波动率影响。高奥、谭娅和吴卫星（2015）[6] 基于上证综合指数构建了度量投资者损失厌恶心理的指 标引入GARCH-M模型，分析不同时期投资者损失厌恶对 收益波动性的影响, 发现损失厌恶是引起股市波动的重要 因素。市进, 郭建变 (2016) [2]根据Bondt和Thaler (1985) 的方法系统剖析了过度反应效应在中国A股市场的存在形 式。杨胜刚和成博（2014）[15]基于贝叶斯决策模型研究 发现市场过度反应是导致股市波动的原因之一。Zhang、 Meng和Zhang (2017) [42] 以基金指数为投资者情绪指标, 用GARCH和EGARCH模型研究投资者情绪与股市波动的 关系。Khatua和Pradhan（2014）[31]构建投资者对信息过 度反应指标, 研究发现过度反应在印度股市的确实存在和 说明了其形成机理。Kumari和Mahakud（2015）[32]使用 GARCH模型并在均值框架中引入了情绪指标, 如风险厌 恶指标、过度反应, 进一步发现投资者情绪能较好预测股 票波动率。Frugier（2016）[29]发现在欧洲市场上，投资 者因为过度乐观而导致股价收益产生了波动不对策的现 象。Labidi和Yaakoubi（2016）[33]投资者情绪纳入描述隐 含市场波动的指标, 发现能更好刻画市场波动性。陈其安 和雷小燕（2017）[3]发现投资者风险厌恶的条件下，以投 资者效用最大化为决策目标, 研究投资者情绪对中国股票 市场波动性的影响机理。贺志芳等（2017）[8]发现投资者 情绪会影响时变风险补偿系数影响股价波动。

综上所述, 以往文献利用行为金融学理论的相关函数 及其细化指标对股市波动性的研究还处于初期阶段, 而且 大多文献只是对股市投资者非理性行为进行实证检验, 但 是把这些行为特征或原因通过量化模型引入股市波动性 的研究还不多, 基于价值函数解释和实证影响机理的文献 更少。

因此, 本文接着行为学者的研究方法, 以行为金融学 的相关理论为理论基础, 将价值函数引入实际模型中进行 研究, 研究框架如下图1。本文将行为金融学中的价值函 数引入EGARCH模型, 进一步研究股票市场波动受到投资 者情绪和投资者特质影响的表现。 


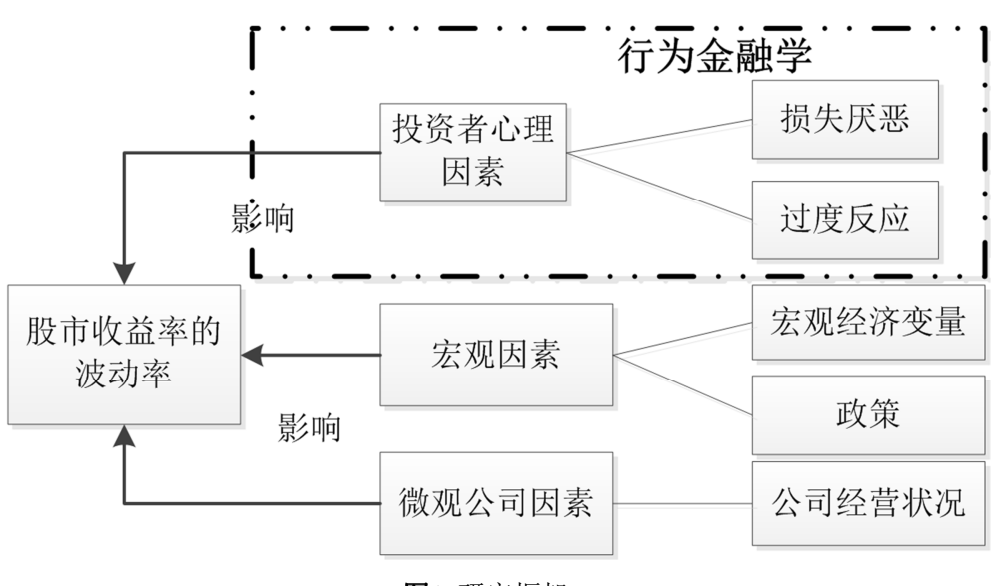

图1 研究框架。

\section{3. 实证检验}

\section{1. 理论假设、度量方式与思路}

现实中投资者在做出复杂的决定时总是表现出非理 性。往往非理性的结果来源于信息处理和行为偏差两个方 面。过度反应是行为偏差的一种体现。过度反应受到研究 者越来越多的重视。其中前景理论(Kahneman和Tversky 1979) 的价值函数体现了投资者心理对于信息的不同反应, 主要体现在损失厌恶, 即面对损失比盈利更加敏感, 同等
程度损失带来的痛苦大于盈利带来的喜悦在股市中, 这种 心理认知偏差会影响到股市的波动性。

除此之外，投资者本身的损失厌恶是人们在决策过程 中，赋予损失的权重远大于收益的权重，使得权重产生偏 差。反应过度也会影响权重, 投资者对信息理解和反应上 会出现偏差导致对损失信息赋权过重, 行为过激。因此, 股价在利空信息下过度下跌而在利好消息下过度上涨。作 用机制如下图 2 , 即损失厌恶、过度反应一一赋权重变化 一市场波动非对称性的逻辑分析框架。

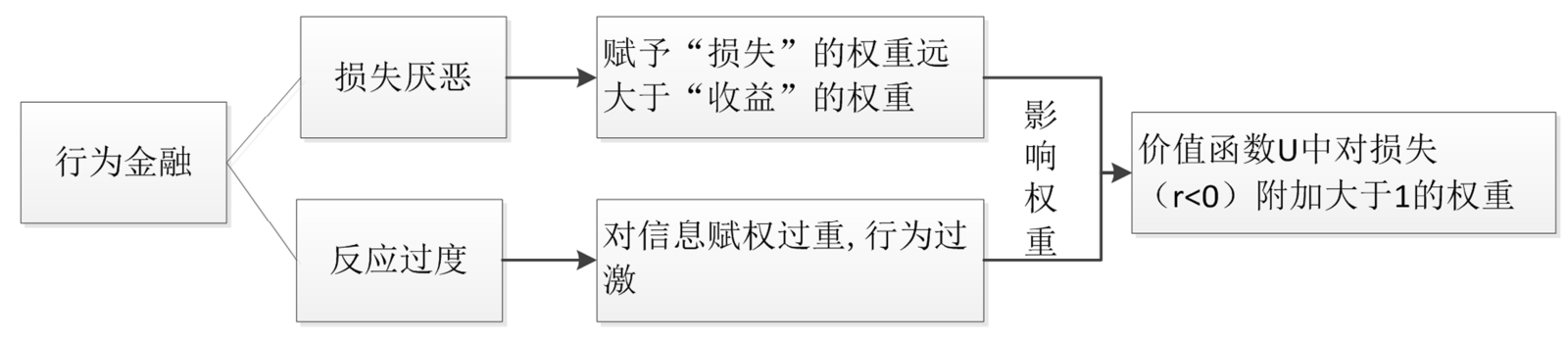

图2 作用机制。

因此提出假设 1 : 损失厌恶和反应过度会导致股票市 场波动呈现非对称性的特点。

根据价值函数，参照Benartzi和Thaler（1995）[24]、 卢斌、王霞（2008）[12]以无风险利率作为参考标准, 考 虑财富的净收益（r) 即财富净收益=财富收入-无风险收 入。此时损失厌恶投资者的效用函数可以表示为:

$$
U(r)=\left\{\begin{array}{c}
r, r_{t} \geq 0 \\
\lambda r, r_{t}<0
\end{array}\right.
$$

同时根据前景理论对损失和收益状态进行区分, 引入 虚拟变量 $\mathrm{S}$ :

$$
S=\left\{\begin{array}{l}
0, r_{t} \geq 0 \\
1, r_{t}<0
\end{array}\right.
$$

公式6中r为投资者遭受的损失或收益的大小。本文通 过参数 $\lambda$ 将收益和损失区别开来。

其中， $\lambda$ 代表损失厌恶系数，即损失带来的负效用是 同等金额的收益所带来的效用的 $\lambda$ 倍, 体现的是偏差带来 的过度反应，会扩大损失结果。本文 $\lambda$ 取值参照Tversky 和Kahneman(1992)大样本调查金融市场的 $\lambda$, 以及卢斌、 王霞（2008）中 $\lambda$ 的取值 $\approx 2.25$, 将原时间序列处理后形成 序列 1 , 进行实证研究。

同时，本文设置对照组， $\lambda=1$ 时即投资者不具有损失 厌恶特征，序列 2 就是原序列，也就是不经过处理的原市 场模型。本文模型采用 $\lambda=2.25$ 和 $\lambda=1$ 分别验证, 并在参数 $\lambda=2.25$ 时引入虚拟变量 $\mathrm{S}$ 。如果参数为 1 的原模型不存在 “利空波动大于利好带来波动”的特性，参数为 2.25 的修正 模型存在“利空大于利好带来波动”的非对称“杜杆效应”, 则体现了损失厌恶对波动非对称性的效应，也就是下图3 成立则推导理论成立, 即能验证假设 1 。 


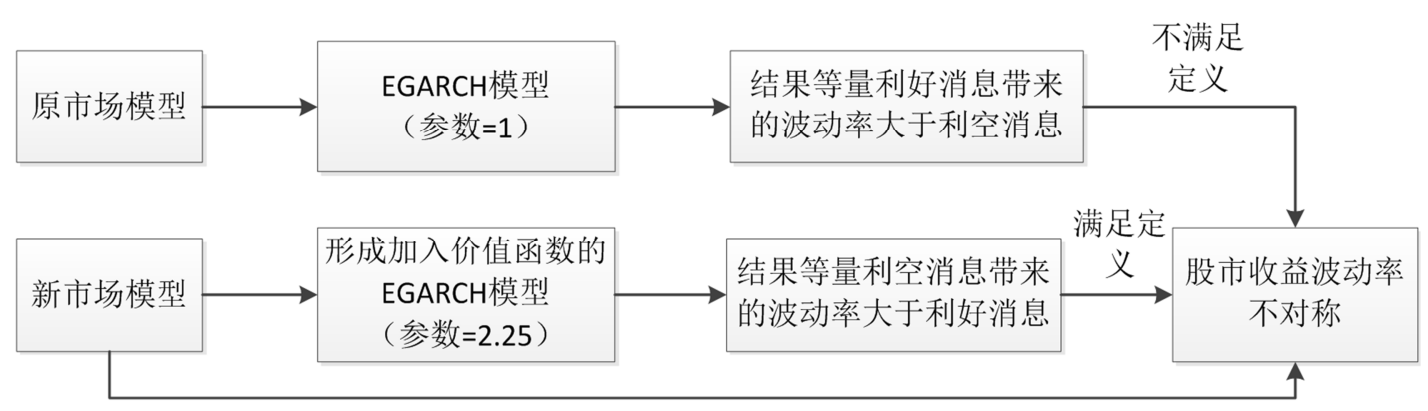

更好刻画, 更好解释

图3 思路推导。

\section{2. 数据选取}

本文选取Wind中A股市场沪深300指数日收盘价, 根据公式8计算对数收益率, 其中 $\mathrm{p}_{\mathrm{t}}$ 表示 $\mathrm{t}$ 时期的收盘价格。 计算得到2008年1月4日至2016年12月31日的日收益率，共2910个观测值。

$$
\mathrm{r}_{\mathrm{t}}=\ln \left(\frac{\mathrm{P}_{\mathrm{t}}}{\mathrm{p}_{\mathrm{t}-1}}\right)
$$

对 $\lambda=1, \lambda=2.25$ 分别建模用 $R$ 语言建模型。（具体步骤如下）

Ires

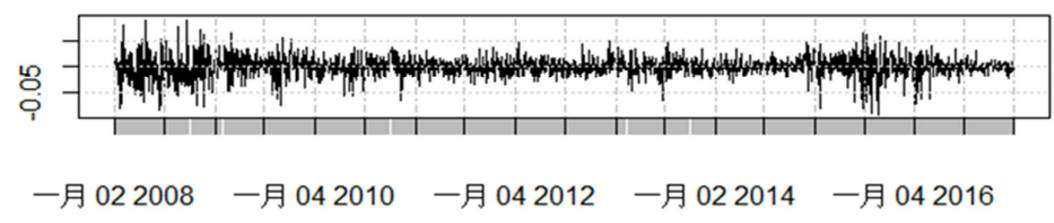

图4 收益率的波动率。

Histogram of Ires

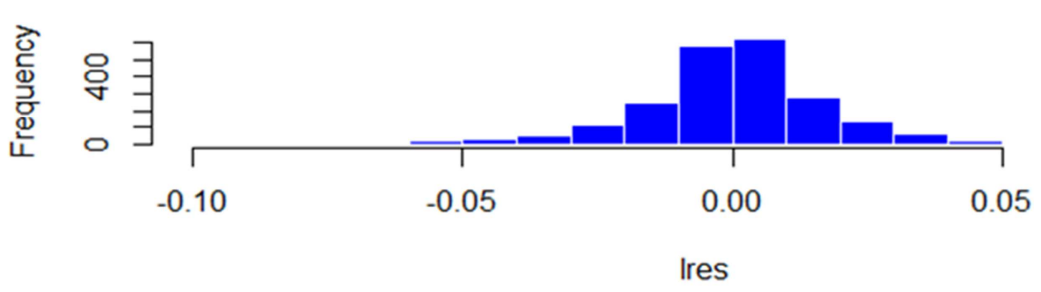

图5 收益率的波动率分布图。

图4、图 5 是以 $\lambda=2.25$ 的序列 1 为例, 收益率的波动率 图与收益率波动率分布图。此时收益率的波动率呈现出非 对称性，说明收益率不是正态分布。与正态分布相比，具 有尖峰厚尾和非对称特征，而且出现波动聚集情况。即当 投资收益在前期表现为向下波动时，投资者在此阶段之后 的财富效用值也会表现为向下波动。

接着本文采用 $\mathrm{LB}$ 检验序列为非白噪声, ADF单位根 检验序列平稳, 进入下一步建模。

\subsection{EGARCH建模}

根据上文分析建立均值方程, 以收益率 $\lambda=2.25$ 的序 列 1 为例, 检查自相关和偏图截尾阶数, 然后尝试AR、 MA、ARMA模型选择最小 AIC 值, 优选得出模型为
$\operatorname{ARMA}(1,4)$, 再根据第三章思路分别对参数 $\lambda=1$ 的序列 1 和 $\lambda=2.25$ 的序列 2 建立E-GARCH模型（见公式 5）。建模 结果如下表1所示:

表1 均值模型。

\begin{tabular}{lllll}
\hline \multirow{2}{*}{ 表题 } & \multicolumn{2}{l}{$\lambda=\mathbf{1}$ (模型1) } & \multicolumn{2}{l}{$\lambda=\mathbf{2 . 2 5}$ (模型2) } \\
\cline { 2 - 5 } & Estimate & $\operatorname{Pr}(>|\mathbf{t}|)$ & Estimate & $\operatorname{Pr}(>|\mathbf{t}|)$ \\
\hline $\mathrm{U}$ & -- & -- & 0.050708 & 0.000000 \\
$\mathrm{~s}$ & -- & - & 0.005095 & 0.000000 \\
omega & -0.093334 & 0.000000 & -0.073585 & 0.000000 \\
alpha1 & -0.025228 & 0.006272 & -0.041705 & 0.000895 \\
beta1 & 0.986892 & 0.000000 & 0.991019 & 0.000000 \\
gamma1 & 0.118049 & 0.000000 & -0.127422 & 0.000542 \\
\hline
\end{tabular}

注: Estimate为估计系数, $\operatorname{Pr}(>\mid t)$ 值为Z统计量的显著程度, 其中反应非 对称项为gamma1估计值系数, 通过判断gamma1系数的正负号, 体现的 是波动性是否具有非对称性。 
根据表 1 可得, $\lambda=1$ (模型 1 ) 和 $\lambda=2.25$ (模型 2 ）的 系数基本都通过了显著性检验。

根据公式 (5) EGARCH模型中的条件方差方程等式 左边是条件方差的对数, 这意味着杜杆影响是指数的, 而 不是二次的, 所以条件方差的预测值一定是非负的。杜杆 效应的存在能够通过 $\gamma<0$ 的得到检验。只要 $\gamma \neq 0$, 冲击的 影响就存在着非对称性, 而且当 $\gamma<0$, 则意味着负冲击比 正冲击更容易增加波动。

$\lambda=1$ 时（左一、左二列）非对称项 gamma1的估计值 大于 0 , 说明等量利好消息比利空消息带来的波动更大, 与非对称效应的定义不匹配, 说明参照组作用成立。

$\lambda=2.25$ 时（右一、右二列）非对称项 gamma1的估计 值小于 0 , 说明存在等量的利空消息产生更大波动。 $\mathrm{U}$ 与 $\mathrm{S}$ 的系数显著且为正, 显示出损失厌恶投资者的效用值与股 市收益率之间相关关系较显著。损失厌恶能反映非对称效
应。可以这样解释：投资者的前期收益为正，投资者的损 失庆恶程度降低, 会适度减少风险规避, 因而大胆买进前 期收益好的股票，使股价格进一步上涨。前期收益为负的 投资者为了避免损失扩大, 大量抛售股票, 致使股票价格 进一步下跌, 解释了波动聚集。同时股票价格稍有上涨时 就勿忙抛出股票套现, 以减少损失, 导致股价格上拉动力 不足, 股价下降的幅度大于股价上涨的幅度, 也解释了非 对称性。

因此本文通过引入行为金融学当中的损失戻恶效应 函数对这种效应存在的原因进行了分析, 从实证的角度发 现非理性投资者的损失厌恶心理偏差是引起股市异常波 动重要因素, 即投资者在股票收益上涨时表现出反应不足, 而在价格下时表现出反应过度。

对比 $\lambda=1$ 时（模型 1 ) 与 $\lambda=2.25$ 时（模型 2 ）的波动率 拟合图, 见下图6。

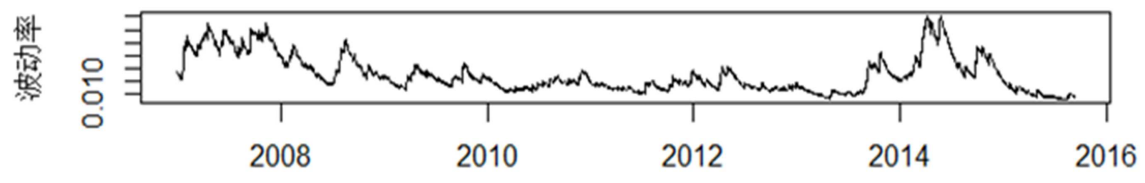

时间

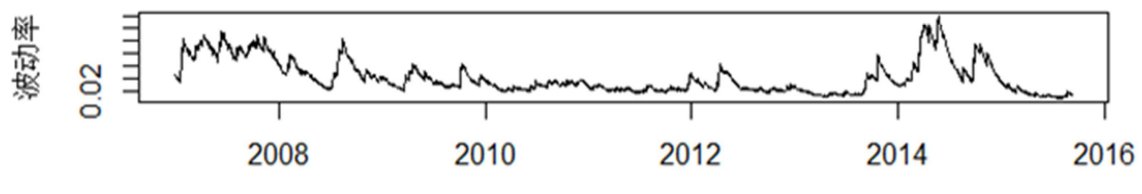

通过对比图6, 修改后的EGARCH模型（模型2）拟合 出提取的波动率比之前的波动率 (模型1) 更为贴近现实, 而原E-GARCH模型 (模型1) 则更为平滑, 与现实不符。 这说明损失厌恶和过度反应确实会导致股票市场收益率 的非对对称性, 因此拟合现实情况更为完美。

\section{4. 行为金融对股市的影响一一波动非对称的影 响}

从前景理论决策角度解释市场非对称波动现象, 相比传 统的解释“波动反馈效应”和“杜杆效应”, 前者更加偏重从更 微观的投资者心理变化和决策行为入手进行推理, 而且通过 上述实证研究也证明了这种解释思路更为合理可信。但是, 投资者情绪和投资者特征也可以理解为是这两种经典解释 的内在原因, 而后者也可以认为是前者的显性表现形式。

从行为金融学对股市波动率的作用上看, 主要通过投 资者损失厌恶的心理、过度反应的行为这两个机制作用在 股市波动率上，使得股市收益的波动率体现出非对称的特 性。股市波动的非对性会带来如下几个方面的影响:

（1）影响风险管理。波动率常用于描述风险, 尤其 是在投资者普遍庆恶风险的情况下，做风险管理时，往往 会计算下行风险、以及 $V a R$ 。常用的方差一协方差法计算
$\mathrm{VaR}$ 的一个关键点是准确预测波动率, 波动率非对称会导 致预测波动率效果不好, 导致风险计算因误差偏离实际值。

(2) 影响股价预测、资产定价产生偏离。正如Antonio Mele(2005)、Jacobs（2016）、Labidi和Yaakoubi (2016) 刘维奇和武翰章（2018）[11]等学者认为投资者非理性情 绪导致定价出现偏离。在非理性的预期下, 投资者要求的 风险补偿是非对称时变的, 所以股票收益的波动率是应该 反经济周期, 也就是说风险补偿在坏时期 (熊市) 比好时 期 (牛市) 所需求的更多。这种情况下波动率的非对称效 应会很大程度上影响资产定价。实际操作上, 投资者通过 波动率研究价格分布, 在掌握标的股票价格波动率特征的 基础上, 利用蒙特卡罗模拟技术对股价路径进行模拟。波 动率非对称的分布会使得价格分布更难模拟, 从而影响股 票市场传导货币政策的财富效应与Q效应。

（3）影响资产的配置。波动率可以做为收益或风险 多因子模型的因子, 比如华泰证券的多因子模型中构建了 收益波动比因子。收益波动率因子组合在股灾期间发生了 较大回撤, 而根据本文实证结果显示, 2015年波动率非对 称非常显著, 可以一定程度上说明非对称波动率会使得原 有策略失去作用。 
再从整个市场俯瞰, 从本质而言, 投资者非理性情绪 和市场过度波动不但增大了市场波动, 高了整体系统性风 险，也不利于营造一个有效合理的市场。

\section{5. 应对策略}

1、一般采用“反转策略”，根据均值回归，通过量化 交易减少人为因素导致的偏差。买进过去表现差的股票而 卖出过去表现好的股票，从中获取超常收益。

2、从本质上避免这些认知错误的方法是减少交易并 实施简单的“购买并持有”策略, 训练有素的行为金融投资 者可以在捕捉到市场价格被错误定价的股票后, 率先集中 资金进行集中投资，赢取更大的收益。

3、投资者在投资过程中详细地记录其要点, 保留长 期的“投资记录”, 多做回测。使其更早地认识并承认这些 认知偏差。

4、对投资者特质、决策偏好特征进行准确刻画。通 过了解投资者情绪、特质和决策偏好特征不仅能够为相关 的理论建模提供基础支持，而且还可以在金融实践中帮助 金融机构制定相应的市场营销和客户服务策略, 为政府决 策部门提供合理的宏观、微观经济政策建议等。

\section{6. 结论}

本文通过理论分析和实证检验结论如下：（1）市场 不对称波动, 可部分归因于投资者本身所具有的损失厌恶、 过度反应特性。市场上投资者情绪水平变化, 通过投资者 损失庆恶的心理、过度反应的行为这两个机制作用在股市 波动率上，使得股市收益的波动率体现出非对称的特性。

(2) 中国 A股市场确实存在过度反应现象。具体表现为引 入损失庈恶效应函数的EGARCH模型结果表明损失庆恶 过度反应的偏差导致存在等量的利空消息产生更大波动 的非对称性，即投资者在股票收益上涨时表现出反应不足， 而在价格下跌时表现出反应过度。

股市收益波动的非对称影响风险管理，非对称导致预测、 描述风险效果不好; 影响资产定价, 非对称股价分布模拟效 果不佳; 影响资产配置, 非对称使得因子模型失去作用。

投资者根据本文结论和投资者情绪、特质多股市波动 性的影响机理, 了解自身的决策偏好, 有利于其作出更为 理性的判断; 对于这种由于损失厌恶、过度反应导致的非 对称波动，投资者可采取反转策略，做好回测，或实施简 单的“购买并持有”策略。

金融机构可以据此立足客户特质提供相应的客户营 销、服务策略。决策和监管部门可以据此制定政策、进行 有效监管，有利于营造一个更为健康的股票市场。

\section{参考文献}

[1] 巴曙松,朱虹.融资融券、投资者情绪与市场波动[J].国际金 融研究,2016(08):82-96。
[2] 市进, 郭建变.股票市场过度反应与反向收益影响因素研究 [J].财经问题研究，2016，(05): 76-82。

[3] 陈其安,雷小燕.货币政策、投资者情绪与中国股票市场波动 性:理论与实证 $[J]$.中国管理科学.2017, (11): 1-11。

[4] 邵振文,侯丹.我国股指期货市场非对称性波动与下行风险 研究[J].经济纵横,2018(03):108-113。

[5]单馨,李旭瑞.投资者情绪与不同规模股票市场指数的关系 研究[J].经济界,2017(05):54-61。

[6] 高奥，谭娅，吴卫星.中国股市收益波动性与投资者损失厌 恶——基于上证综合指数的实证研究 [J]. 南方金融, 2015, (01):62-73。

[7] 贺刚,朱淑珍,顾海峰.中美股市的“创造空间效应”与“弗里德 曼效应”比较分析: 基于投资者情绪波动影响视角 $[\mathrm{J}]$. 世界经 济研究,2017(10):34-44+135。

[8] 贺志芳,文风华,黄创霞,杨晓光,郑石明.投资者情绪与时变 风险补偿系数[J].管理科学学报,2017,20(12):29-38。

[9] 胡昌生,池阳春. 投资者情绪、资产估值与股票市场波动 [J]. 金融研究.2013，(10)：181-193。

[10] 刘璐, 张倩.亚洲地区股票指数收益率的波动性研究——基 于 GARCH 族模型[J]. 区域金融研究，2011，(01):34-39。

[11] 刘维奇,武翰章.投资者情绪会影响股票市场的误定价 吗? 一一基于上证 $\mathrm{A}$ 股市场的实证研究 [J]. 金融与经 济,2018(03):19-25。

[12] 卢斌, 王霞.中国股票市场波动非对称性的行为金融学解释 [J].南京财经大学学报, 2008, (06):36-41。

[13] 陆静,周媛.投资者情绪对股价的影响——基于 $\mathrm{AH}$ 股交叉 上市股票的实证分析 [J].中国管理科学,2015,23(11):21-28。

[14] 文凤华,肖金利,黄创霞,陈晓红,杨晓光.投资者情绪特征对 股票价格行为的影响研究 [J]. 管理科学学 报,2014,17(03):60-69。

[15] 杨胜刚,成博.基于贝叶斯决策的证券市场过度反应 [J].系统 工程,2014,32(10):24-29。

[16] 翟佳茹.基于 GRACH 类模型的法兰克福 DAX 指数收益率 波动性研究 [J].企业改革与管理,2017(15):104-105。

[17] 张普,陈亮,曹启龙.信息视角下基于异质信念的股票波动性 价值研究 [J].管理科学,2018,31(02):147-160。

[18] 张宗新, 王海亮.投资者情绪、主观信念调整与市场波动 $[\mathrm{J}]$. 金融研究, 2013，(04): 142-155。

[19] 郑伟伦.上证综合指数股票价格短期预测——基于 ARIMA 模型的研究分析 [J]. 经济研究导刊, 2014, (16):136-13。

[20] 朱钧钧, 谢识予.中国股市波动率的双重不对称性及其解释 —基于 MS-TGARCH 模型的 MCMC 估计和分析[J]. 金融 研究, 2011, (03): 134-148。

[21] 朱超,曹洳宜.投资者非理性情绪、分析师评级变动与股票市 场波动 $[\mathrm{J}]$.金融科学,2017(02):58-76。 
[22] Baek C. Stock prices, dividends, earnings, and investor sentiment $[\mathrm{J}]$. Review of Quantitative Finance \& Accounting, 2016, 47(4):1043-1061.

[23] Benartzi S., R. H. Thaler. Myopic Loss Aversion and the Equity Premium Puzzle[J].The Quarterly Journal of Economics, 1995, 110(1).

[24] Bondt,W.F.M.D.,Thaler,R. Does The Stock Market Overreact? [J]. The Journal of Finance, 1985,40(3):793-805.

[25] Bollerslev T. Generalised Autoregressive Conditional Heteroskedasticity[J]. Econometrica, 1986, 31(302):2-27.

[26] Box G E P, Jenkins G M, Reinsel G C. Time Series Analysis, Fourth Edition [M].Time Series Analysis. 2013:137-191.

[27] Chandra A, Thenmozhi M. Investor Sentiment, Volatility and Stock Return Comovements[J]. Ssrn Electronic Journal, 2013.

[28] Engle R F. Autoregressive Conditional Heteroscedasticity with Estimates of the Variance of United Kingdom Inflation[J]. Econometrica, 1982, 50(4):987-1007.

[29] Frugier A. Returns, volatility and investor sentiment: Evidence from European stock markets[J]. Research in International Business \& Finance, 2016, 38:45-55.

[30] Jacobs H. Market maturity and mispricing [J]. Journal of Financial Economics, 2016, 122(2):270-287.

[31] Khatua S, Pradhan H K. Examining Overreaction in Indian Stock Market for Quarterly News[J]. Emaj Emerging Markets Journal, 2014, 4(1).

[32] Kumari J, Mahakud J. Does investor sentiment predict the asset volatility? Evidence from emerging stock market India[J]. Journal of Behavioral \& Experimental Finance, 2015, 8:25-39.

[33] Labidi C, Yaakoubi S. Investor sentiment and aggregate volatility pricing $[\mathrm{J}]$. Quarterly Review of Economics \& Finance, 2016, 61:53-63.
[34] Sayim M, Morris P D, Rahman H. The effect of US individual investor sentiment on industry - specific stock returns and volatility[J]. Review of Behavioral Finance, 2013, 5(1):58-76.

[35] Spyros Spyrou. Sentiment changes, stock returns and volatility: evidence from NYSE, AMEX and NASDAQ stocks[J]. Applied Financial Economics, 2012, 22(19):1631-1646.

[36] Schneller D, Heiden S, Heiden M, et al. Home is Where You Know Your Volatility - Local Investor Sentiment and Stock Market Volatility[J]. German Economic Review, 2017.

[37] Tversky A, Kahneman D. Advance in Prospect Theory: Cumulative Representation of Uncertainty [J]. Journal of Risk and Uncertainty, 1992, 5: 297-323.

[38] TC Chiang, CWS Chen, MKP So Asymmetric Return and Volatility Responses to Composite News from Stock Markets, [J].Multinational Finance Journal，2007， 11 (3-4):179-210.

[39] Uygur U, Taş O. The impacts of investor sentiment on returns and conditional volatility of international stock markets[J]. Quality \& Quantity, 2014, 48(3):1165-1179.

[40] Walid Saleh. Overreaction: the sensitivity of defining the duration of the formation period[J]. Applied Financial Economics. 2007, (1):45-61.

[41] Yang K. Investor Sentiment and the Short-term Volatility of Stock Market:on What Happened in China in 2015[J]. Future \& Development, 2016.

[42] Zhang W Y, Meng N D, Zhang T P. Research on the Stock Market Volatility Influenced by Institutional Investor in China: Based on Investor Sentiment[J]. Journal of Beijing University of Technology, 2017. 